\title{
A comparative study of efficacy of 1064-nm Q-switched Neodymium: YAG laser at sub photothermolytic fluence alone and in combination therapy with epicutaneous $20 \%$ azelaic acid and in sequential therapy with microdermabrasion in melasma in South India
}

\author{
Samuel J. Daniel ${ }^{1}$, Ramalingam S. Kumar, ${ }^{2, *}$, Kuppuswamy P. Geetha ${ }^{3}$ \\ ${ }^{\mathbf{1}}$ Associate Professor, ${ }^{2}$ Assistant Professor, ${ }^{3}$ Junior Resident, Dept. of Dermatology, Government Thiruvarur Medical College, \\ Thiruvarur, Tamil Nadu, India \\ *Corresponding Author: \\ Email: sureshkumargtmc@gmail.com
}

\begin{abstract}
Introduction: Melasma is a symmetric hyperpigmentary disorder defined by irregular light to brown macules over the face. Treating melasma is demanding and there is curiosity in anticipation of recent various therapeutic options like sub photothermolytic fluence - 1064-nm Q switched Nd:YAG laser (SPF-1064-nm-QSNdYL) with combinational and sequential therapies.

Aim: To study and compare the therapeutic effect of SPF-1064-nm-QSNdYL alone and in combinational therapy with epicutaneous 20\% Azelaic Acid and in sequential therapy with microdermabrasion in melasma in three study groups of 20 cases each.

Materials and Methods: Sixty female cases identified as melasma were randomly segregated into three factions [group L(laser only) $=20$ cases of melasma treated with SPF-1064-nm-QSNdYL at weekly intervals, group C (combinational) $=20$ cases of melasma treated with twice daily epicutaneous application of $20 \%$ azelaic acid and SPF-1064-nm-QSNdYL at weekly intervals and group $\mathrm{S}$ (sequential) = 20 cases of melasma treated with Sequential therapy with microdermabrasion followed by SPF-1064$\mathrm{nm}$-QSNdYL once weekly as given in group L]. The study duration was of 12 weeks for each. The outcome to therapy was analyzed using melasma area and severity index scoring.

Results: Convincing therapeutic response was noted in all the three factions. It was statistically highly significant in Group $\mathrm{C}$ as compared to Group L $(\mathrm{P}<0.001)$ and Group $\mathrm{S}(\mathrm{P}<0.001)$. However no statistically significant difference was noted in the reduction of MASI scoring between the Group L and Group $\mathrm{S}(\mathrm{P}>0.05)$.

Conclusions: This study highlights the superior therapeutic effect of combinational therapy using SPF-1064-nm- QSNdYL with azelaic acid in epidermal, dermal and mixed types of melasma with minimal adverse effects followed by sequential therapy of microdermabrasion with SPF-1064-nm- QSNdYL and SPF-1064-nm- QSNdYL alone therapy.
\end{abstract}

Keywords: Azelaic acid, SPF-1064-nm-QSNdYL, Microdermabrasion, Melasma.

\section{Introduction}

Melasma is a commonly acquired circumscribed hyperpigmentary disorder characterized by poorly defined, symmetric, hyperpigmented patches with an irregular outline mainly involving the sun exposed areas especially the face. It is most prevalent among young to middle-aged women specifically in dark skin toned races that habitat in areas of intense UV rays like Asians, Blacks and Latin Americans. ${ }^{1}$ Just $10 \%$ of cases were noted in males. In India, the incidence is only $10 \%$. The recognized etiological factors that leads to melasma are pregnant status, using cosmetical agents, genetic effects, endocrinological effects, microvascular effects and hormone treatment. ${ }^{2-4}$ The above mentioned factors augment the melanosomal synthesis in the melanocytes and also an enhanced transport of melanosomes to keratinocytes. ${ }^{5}$ Though melasma is benign disorder there are frequent relapses and therapeutic resistance that causes immense emotional and psycho-social hassle in both males and females disrupting their quality of life.

Three clinical morphology of melasma are Centrofacial (most common), malar and mandibular. ${ }^{6}$
On the basis of visible light, Wood's lamp and dermatopathology, melasma can be divided into three clinical variants. Epidermal variant with an increase in melanin predominantly in the basal and suprabasal layers of the epidermis with accentuated pigment enhancement on examination with Wood's lamp. The dermal variant with melanin-laden macrophages distributed perivascularly in the superficial and deep dermal layers without pigmentary accentuation on Wood's lamp. The mixed variant with elements of both appearing as deep brown in colour with Wood's lamp. Only the epidermal variant shows pigment accentuation.., 7

The management of melasma has been a paradox specifically in dark coloured patients. The limitation is to reduce the pigmention of melasma without the expected complications like hypomelanosis or postinflammatory hypermelanosis. Hence it necessitates the protection from high intense UV rays by the use of broad spectrum sunscreens, be underneath shade if accessible and to use protective head covers and clothes. Standard treatment protocols used in melasma with some therapeutic effects are topical depigmentary 
medications like hydroquinone, kojic acid and azelaic acid frequently used by combining with other agents like topical corticosteroids, tretinoin and chemical peels. Of late, epicutaneous azelaic acid is an efficient and well tolerable therapeutic option for hyperpigmentary disorders specifically in dark coloured patients. Ongoing advances in laser research has enhanced armory of dermatological therapeutics to use lasers for selective removal of pigmentation.

In melasma the prior use of conventional highfluence QSNdYL was indeed effective in removal of pigmentation but at the cost of increased risk of postinflammatory hypermelanosis in some cases. ${ }^{8}$ This study establishes the efficacy and safe profile of 1064$\mathrm{nm}$ QSNdYL alone and in combinational therapy with epicutaneous 20\% Azelaic acid and in sequential therapy with microdermabrasion in melasma in all the three study factions of 20 cases each. Clinical assessment was done by taking into account the reduction in pigmentation and as well the area of pigment distribution by melasma area and severity index (MASI) score. ${ }^{9}$

\section{Materials and Methods}

Sixty female cases diagnosed as melasma and not undergone any treatment prior were admitted in this study. They were randomly segregated into three factions [group L (Laser only) $=20$ cases who underwent sub photothermolytic fluence - 1064-nm Q switched Nd:YAG laser(SPF-1064-nm-QSNdYL) at weekly intervals, group C (combinational therapy) $=20$ cases were prescribed with twice daily application of $20 \%$ azelaic acid with SPF-1064-nm-QSNdYL at weekly intervals and group $S$ (sequential therapy) $=20$ cases were given Sequential therapy with microdermabrasion followed by SPF-1064-nmQSNdYL once weekly as advised in group L]. All cases attending our outpatient department of Dermatology in our hospital were enlisted for a study duration of 12 weeks. Women aged 18 and above with Fitzpatrick skin types III, IV, \& V having diagnosed as melasma and not undergone any treatment so far were enlisted in this research except those over 60 years of age. Only those subjects who were regular were admitted. Patients on oral contraception medications, hormone replacement treatments, women with pregnacy and lactation, acute infectious diseases, photosensitivity and cases with systemic or endocrine disorders or hypersensitive response to topical azelaic acid or topical anaesthetic medications were not enrolled in this study. The patients were interviewed in person after obtaining informed written consent. Initially a detailed history was taken regarding the age of occurrence, duration of pigmentation, occupation, family history, relation to pregnancy, drug intake in the form of OCP, antiepileptics and any other precipitating factors.

Melasma was diagnosed as epidermal, dermal or mixed clinically, under wood's lamp and also by histopathology in cases that consented for skin biopsy. Before the study commenced, approval by the Institutional ethical committee was obtained. Information on sunlight safeguard and topical application of a broad spectrum sunscreen medications (of SPF > 30) during the study duration was provided to the patients. Photographs were documented at 0 week (when study started), at 6 weeks (during study period) and at 12 weeks (when therapy finished). Epicutaneous anesthesia with eutectic combinations of lignocaine and prilocaine was advised for occlusive application for 2 hours before laser therapy. The Laser hand piece was held perpendicularly to skin surface during procedure with minimum overlaping. The whole area under treatment was covered by a single pass. Appropriate eye protecting goggles was provided to all those who were inside according to safety protocol during the laser procedure. All the patients who underwent the procedure wore a silicone coated lead covers as advised by the laser manufacturing company.

Cases in group L were treated with SPF-1064-nmQSNdYL at parameters of 6-8 mm spot size, $10 \mathrm{~Hz}$, $0.5-1 \mathrm{~J} / \mathrm{cm} \mathrm{2}$, at 10 passes per week for a total duration of 12 weeks. Weekly incremental dosage of $0.1 \mathrm{~J}$ was administered until attaining an energy fluence of $1 \mathrm{~J} / \mathrm{cm}$ 2 and it was further continued at the same dose till the end of 12 weeks. The end point was attained when instant lightening effect or erythematous skin lesions was noted. Cases in group $\mathrm{C}$ were treated with a combinational therapy of both, SPF-1064-nm-QSNdYL once weekly as given in group $\mathrm{L}$ and epicutaneous azelaic acid $20 \%$ applied twice a day over the pigmented area for a total period of 12 weeks leave alone on the morning of laser administration.

Cases in group $\mathrm{S}$ were given sequential therapy with microdermabrasion followed by SPF-1064-nmQSNdYL with fluence of $1.6-2.5 \mathrm{~J} / \mathrm{cm} 2$ at $10 \mathrm{~Hz}$ with a $5-6 \mathrm{~mm}$ spot, using only 2 laser passes to achieve complete coverage of the affected skin, and was administered immediately after microdermabrasion. The same procedure was done at weekly intervals for a period of 12 weeks. Mild erythema was taken as end point.

All the enlisted sixty cases that finished the study were assessed by clinical observation and by evaluating photographs taken serially at the beginning and after every laser sittings. The reduction in intensity as well as the area of pigmentation was graded using Melasma Area and Severity Index, MASI I (at 0 weeks) and MASI II (at12 weeks) for all cases. P value of $<0.05$ was noted as significant and $\mathrm{P} \leq 0.001$ as highly significant. Cases were also noted for any complications during the study period. The statistical analysis was assessed by using chi square test, paired and unpaired student $t$-tests. 


\section{Results}

Sixty female cases from the outpatient department of age group 18 years and above, diagnosed clinically as melasma and not undergone any treatment prior were enlisted in the study. The mean age was $33.6 \pm 6.34$. At the inception of this research the minimum age documented was 19 years and the maximum age was 57 years. Many were in the age ranging from 31 to 40 years. Among them 5 cases were in the age range of 2130 years, 37 in the age range of $31-40$ years and 17 were greater than 41 years of age (Table 1). Also 16 (26.66\%) cases were of phototype IV and $44(73.34 \%)$ cases were of photo type V. All the three factions had no statistically significant differences in the age group range, skin phototyping and morphological patterns of melasma on comparison. The time span of melasma varied from 4 months to 16 years and the mean duration of the disease was 4.6 years. Major precipitating factor in $38(63.33 \%)$ patients was sun exposure followed by positive family history in $23(38.33 \%)$ patients, 4 $(6.66 \%)$ patients had associated thyroid disorders and in $2(3.33 \%)$ patients were attributed to drug. Onset of melasma was not associated with pregnant status in $45.6 \%$ of cases.

Centero facial type of melasma was the most commonly noted $(70 \%)$, followed by malar $(21.66 \%)$ and the mandibular type in $8.34 \%$ of cases. The distribution of clinical types of melasma in each faction is noted in Table 2. Under woods lamp, epidermal type was noted in 7(11.67\%), dermal type in $12(20 \%)$ and mixed type was seen in 41 (68.33\%) patients (Table 3). Out of the three patients who consented for skin biopsy, clinical and woods lamp examination correlated with histopathological finding in all three cases (two were mixed type and one was epidermal type).

The Mean baseline MASI scoring (MASI I) of group L was $15.35 \pm 6.24$, group C $17.42 \pm 6.89$ and group $S$ was $20.67 \pm 5.37$. On comparison no statistically significant differences was seen among the groups ( $P$ $>0.05$ ). After 12 weeks of completing the study, the mean MASI scoring (MASI II) among three factions were 9.26 \pm 3.68 in group L, $4.36 \pm 1.84$ in group $C$ and $11.58 \pm 2.65$ in Group $S$ (Table 4). Statistically significant improvement $(\mathrm{P}<0.05)$ was noted in all the three factions after therapy. The clinical outcome in
Groups L(laser alone), C (combinational therapy) and S (sequential therapy) are shown in Fig. 1-6.

The reduction in the MASI scoring after completing the study was maximum in group $\mathrm{C}$ [combinational therapy of sub photothermolytic fluence - 1064-nm Q switched Nd:YAG laser(SPF-1064-nmQSNdYL) and 20\% azelaic acid] followed by group S [sequential therapy of microdermabrasion with SPF1064-nm-QSNdYL] and group L(SPF-1064-nmQSNdYL alone therapy). This improvement was statistically highly significant in group $\mathrm{C}$ when compared to group $\mathrm{L}(\mathrm{P}<0.001)$ and group $\mathrm{S}(\mathrm{P}<$ $0.001)$. However the differences in reduction of MASI scoring between the group $\mathrm{L}$ and group $\mathrm{S}$ was not statistically significant $(\mathrm{P}>0.05)$. (Table 5)

All the three methods of therapy showed a significant decrease in mean MASI scoring after 12 weeks in all clinical types of melasma. However on comparison, in epidermal melasma type, statistically significant results were noted in group $\mathrm{C}$ when related to group $\mathrm{L}(\mathrm{P}<0.05)$ and group $\mathrm{S}(\mathrm{P}<0.05)$. But no significant statistical differences were noted in the reduction of MASI scoring between group L and Group $\mathrm{S}(\mathrm{P}>0.05)$. In dermal type of melasma, statistically significant differentiation in results was noted in both group $\mathrm{C}$ and group $\mathrm{S}$ on comparing to group $\mathrm{L}(\mathrm{P}<$ 0.05 ). However not much statistically significant differentiation in reduction of MASI scoring was noted between group $\mathrm{C}$ and group $\mathrm{S}(\mathrm{P}>0.05)$. Group $\mathrm{C}$ showed a statistically significant improvement in mixed melasma when compared to group L $(\mathrm{P}<0.001)$ and group $\mathrm{S}(\mathrm{P}<0.001)$. (Table 6)

No complications were noted in Group L (SPF1064-nm-QSNdYL). In Group C (combinational therapy of SPF-1064-nm-QSNdYL and 20\% azelaic acid), 2 (10\%) patients had mild burning sensation and $1(5 \%)$ patient had transient erythema. In group S (sequential therapy of microdermabrasion with SPF1064-nm- QSNdYL), postinflammatory hyperpigmentation developed in $3(15 \%)$ patients, erythema in $2(10 \%)$ patients (Fig. 7) and only $1(5 \%)$ patient had burning sensation. All these complications except post inflammatory hyperpigmentation were mild and decreased within an hour in all the cases.

Table 1: Distribution of melasma in each study faction with emphasis on age $(n=60)$

\begin{tabular}{|c|c|c|c|c|}
\hline $\begin{array}{c}\text { Age group } \\
(\text { years })\end{array}$ & $\begin{array}{c}\text { Group } \mathbf{c} \\
(\mathbf{n = 2 0}) \\
\text { No. of cases }(\%)\end{array}$ & $\begin{array}{c}\text { Group C } \\
(\mathbf{n = 2 0}) \\
\text { No. of cases }(\%)\end{array}$ & $\begin{array}{c}\text { Group } \mathbf{~} \\
(\mathbf{n}=\mathbf{2 0}) \\
\text { No. of cases(\%) }\end{array}$ & $\begin{array}{c}\text { Total } \\
(\mathbf{n}=\mathbf{6 0}) \\
\text { No. of cases(\%) }\end{array}$ \\
\hline$<20$ & & $1(5)$ & & $1(1.67)$ \\
\hline $21-30$ & $2(10)$ & $2(10)$ & $1(5)$ & $5(8.30)$ \\
\hline $31-40$ & $11(55)$ & $13(65)$ & $13(65)$ & $37(61.66)$ \\
\hline$>41$ & $7(35)$ & $4(20)$ & $6(30)$ & $17(28.33)$ \\
\hline Total $(\%)$ & $20(100)$ & $20(100)$ & $20(100)$ & $60(100)$ \\
\hline
\end{tabular}


Table 2: Distribution of melasma in each study faction with emphasis on clinical pattern(n=60)

\begin{tabular}{|c|c|c|c|c|}
\hline $\begin{array}{l}\text { Clinical } \\
\text { Pattern }\end{array}$ & $\begin{array}{c}\text { Group L } \\
(n=20) \\
\text { No. of cases }(\%) \\
\end{array}$ & $\begin{array}{c}\text { Group C } \\
(\mathbf{n}=\mathbf{2 0}) \\
\text { No. of } \operatorname{cases}(\%) \\
\end{array}$ & $\begin{array}{c}\text { Group S } \\
(\mathbf{n}=\mathbf{2 0}) \\
\text { No. of cases }(\%) \\
\end{array}$ & $\begin{array}{c}\text { Total } \\
(n=60) \\
\text { No. of cases }(\%)\end{array}$ \\
\hline Centero facial & $12(60)$ & $14(70)$ & $16(80)$ & $42(70)$ \\
\hline Malar & $6(30)$ & $4(20)$ & $3(15)$ & $13(21.66)$ \\
\hline Mandibular & $2(10)$ & $2(10)$ & $1(5)$ & $05(8.34)$ \\
\hline Total $(\%)$ & $20(100)$ & $20(100)$ & $20(100)$ & $60(100)$ \\
\hline
\end{tabular}

Table 3: Distribution of melasma in each study faction under Wood's $\operatorname{Lamp}(n=60)$

\begin{tabular}{|l|l|l|l|l|}
\hline $\begin{array}{c}\text { Clinical } \\
\text { Pattern }\end{array}$ & \multicolumn{1}{|c|}{$\begin{array}{c}\text { Group L } \\
(\mathbf{n = 2 0}) \\
\text { No. of cases }(\%)\end{array}$} & $\begin{array}{c}\text { Group C } \\
(\mathbf{n = 2 0}) \\
\text { No. of cases }(\%)\end{array}$ & $\begin{array}{c}\text { Group S } \\
(\mathbf{n = 2 0}) \\
\text { No. of cases }(\%)\end{array}$ & $\begin{array}{c}\text { Total } \\
(\mathbf{n = 6 0}) \\
\text { No. of cases(\%) }\end{array}$ \\
\hline Epidermal & $2(10)$ & $1(5)$ & $4(20)$ & $7(11.67)$ \\
\hline Dermal & $5(30)$ & $4(20)$ & $3(15)$ & $12(20)$ \\
\hline Mixed & $13(70)$ & $15(75)$ & $13(65)$ & $41(68.33)$ \\
\hline Total $(\%)$ & $20(100)$ & $20(100)$ & $20(100)$ & $60(100)$ \\
\hline
\end{tabular}

Table 4: MASI scoring at baseline and after 12 weeks treatment $(n=60)$

\begin{tabular}{|l|c|c|c|c|}
\hline \multicolumn{1}{|c|}{ Group } & $\begin{array}{c}\text { MASI - I } \\
\text { (at baseline) }\end{array}$ & $\begin{array}{c}\text { MASI - II } \\
\text { (after 12 weeks) }\end{array}$ & $\begin{array}{c}\text { Improvement } \\
(\mathbf{\%})\end{array}$ & P value \\
\hline $\begin{array}{l}\mathrm{L} \\
(\mathrm{n}=20)\end{array}$ & $15.35 \pm 6.24$ & $9.26 \pm 3.68$ & 39.67 & $<0.05$ \\
\hline $\begin{array}{l}\mathrm{C} \\
(\mathrm{n}=20)\end{array}$ & $17.42 \pm 6.89$ & $4.36 \pm 2.84$ & 74.96 & $<0.05$ \\
\hline $\begin{array}{l}\mathrm{S} \\
(\mathrm{n}=20)\end{array}$ & $20.67 \pm 5.37$ & $11.58 \pm 2.65$ & 43.97 & $<0.05$ \\
\hline
\end{tabular}

Table 5: Comparison of mean melasma area and severity index (MASI) among group L, group C and group $\mathrm{S}$ at day 0,6 and 12 weeks

\begin{tabular}{|c|c|c|c|c|c|c|}
\hline Group & $\begin{array}{l}\text { Mean MASI } \\
\text { 0 weeks }\end{array}$ & $P$ value & $\begin{array}{c}\text { Mean MASI } \\
6 \text { weeks }\end{array}$ & $P$ value & $\begin{array}{c}\text { Mean } \\
\text { MASI } \\
12 \text { weeks }\end{array}$ & $P$ value \\
\hline $\mathrm{L}$ & $15.35 \pm 6.24$ & \multirow[t]{2}{*}{0.173} & $12.39 \pm 4.65$ & \multirow[t]{2}{*}{0.136} & $9.26 \pm 3.68$ & \multirow[t]{2}{*}{0.000} \\
\hline $\mathrm{C}$ & $17.42 \pm 6.89$ & & $10.08 \pm 4.32$ & & $4.36 \pm 1.84$ & \\
\hline $\mathrm{L}$ & $15.35 \pm 6.24$ & \multirow[t]{2}{*}{0.068} & $12.39 \pm 4.65$ & \multirow[t]{2}{*}{0.089} & $9.26 \pm 3.68$ & \multirow[t]{2}{*}{0.743} \\
\hline$S$ & $20.67 \pm 5.37$ & & $14.43 \pm 5.14$ & & $11.58 \pm 2.65$ & \\
\hline $\mathrm{C}$ & $17.42 \pm 6.89$ & \multirow[t]{2}{*}{0.316} & $10.08 \pm 4.32$ & \multirow[t]{2}{*}{0.165} & $4.36 \pm 1.84$ & \multirow[t]{2}{*}{0.000} \\
\hline$S$ & $20.67 \pm 5.37$ & & $14.43 \pm 5.14$ & & $11.58 \pm 2.65$ & \\
\hline
\end{tabular}

Table 6: Comparison among groups $\mathrm{L}, \mathrm{C}$ and $\mathrm{S}$ in melasma area and severity index (MASI) scoring at 0,6,12 weeks with emphasis on the morphology of melasma (epidermal, dermal and mixed)

\begin{tabular}{|l|c|c|c|}
\hline Type of Melasma & MASI - O (mean \pm SD) & MASI - 6(mean \pm SD) & MASI -12(mean \pm SD) \\
\hline Epidermal melasma & $18.69 \pm 3.03$ & $14.88 \pm 3.48$ & $11.46 \pm 2.55$ \\
Group L & $20.13 \pm 8.95$ & $13.28 \pm 6.07$ & $5.12 \pm 1.94$ \\
Group C & $27.24 \pm 7.84$ & $20.29 \pm 4.11$ & $15.91 \pm 1.04$ \\
Group S & $>0.05$ & $>0.05$ & 0.043 \\
$P$ value (L and C) & $>0.05$ & 0.003 & 0.015 \\
$P$ value (C and S) & $>0.05$ & $>0.05$ & 0.832 \\
$P$ value (L and S) & $14.12 \pm 5.98$ & $11.96 \pm 4.82$ & $7.64 \pm 3.59$ \\
\hline Dermal melasma & $17.51 \pm 8.96$ & $10.03 \pm 6.69$ & $5.20 \pm 2.77$ \\
Group L & $16.57 \pm 8.45$ & $12.26 \pm 5.68$ & $6.42 \pm 1.08$ \\
Group C & $>0.05$ & 0.003 & 0.000 \\
Group S & $>0.05$ & 0.028 & 0.302 \\
$P$ value (L and C) & $>0.05$ & 0.145 & 0.000 \\
$P$ value (C and S) & & & \\
$P$ value (L and S) & & & \\
\hline
\end{tabular}




\begin{tabular}{|l|c|c|c|}
\hline Mixed melasma & $15.41 \pm 6.78$ & $12.19 \pm 7.34$ & $8.67 \pm 3.94$ \\
Group L & $16.94 \pm 6.53$ & $9.41 \pm 3.98$ & $4.08 \pm 1.79$ \\
Group C & $20.18 \pm 4.29$ & $14.23 \pm 4.36$ & $10.74 \pm 1.48$ \\
Group S & 0.00 & $<0.001$ \\
$P$ value (L and C) & & 0.00 & $<0.001$ \\
$P$ value $(\mathrm{C}$ and S) & & 0.128 & $<0.001$ \\
$P$ value (L and S) & & & \\
\hline
\end{tabular}

Table 7: Complications in each study group $(n=60)$

\begin{tabular}{|l|c|c|c|c|}
\hline \multicolumn{1}{|c|}{ Complications } & $\begin{array}{c}\text { Group } \mathbf{L} \\
(\mathbf{n}=\mathbf{2 0}) \\
\text { No. of cases(\%) }\end{array}$ & $\begin{array}{c}\text { Group C } \\
(\mathbf{n}=\mathbf{2 0}) \\
\text { No. of cases(\%) }\end{array}$ & $\begin{array}{c}\text { Group S } \\
(\mathbf{n = 2 0}) \\
\text { No. of cases(\%) }\end{array}$ & $\begin{array}{c}\text { Total } \\
(\mathbf{n = 6 0}) \\
\text { No. of cases(\%) }\end{array}$ \\
\hline Erythema & 0 & $1(5)$ & $2(10)$ & $3(5)$ \\
\hline PIH & 0 & 0 & $3(15)$ & $3(5)$ \\
\hline Burning sensation & 0 & $2(10)$ & $1(5)$ & $3(5)$ \\
\hline Nil & $20(100)$ & $17(85)$ & $14(70)$ & $51(85)$ \\
\hline Total (\%) & $20(100)$ & $20(100)$ & $20(100)$ & $60(100)$ \\
\hline
\end{tabular}

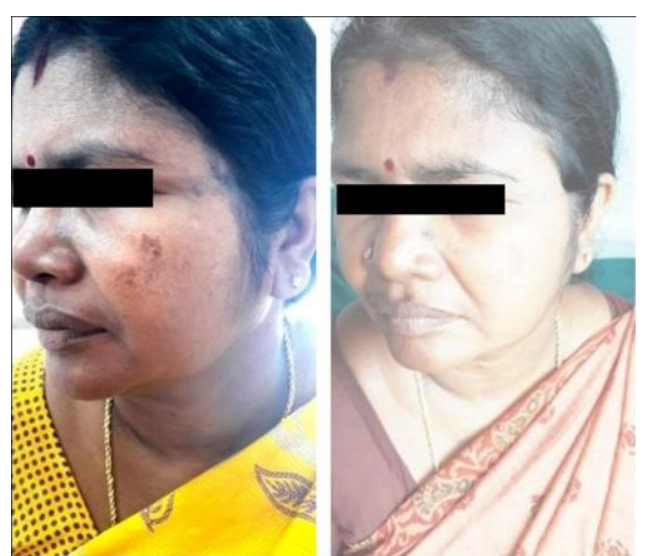

Fig. 1: Photograph showing a case of melasma on SPF-1064-nm-QSNdYL alone therapy. i) week 0 (MASI 13.8) ii) week 12 (MASI 6.3)

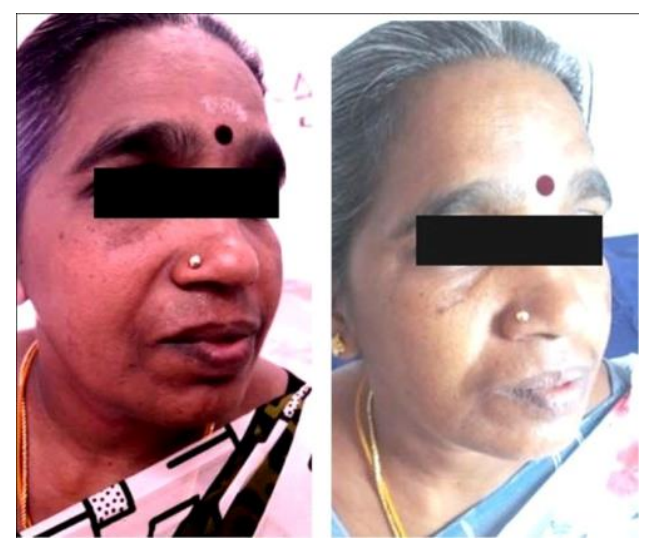

FiG. 2: Photograph showing a case of melasma on SPF-1064-nm-QSNdYL alone therapy.

i) week 0 (MASI 9.7); ii) week 12 (MASI 6.2)

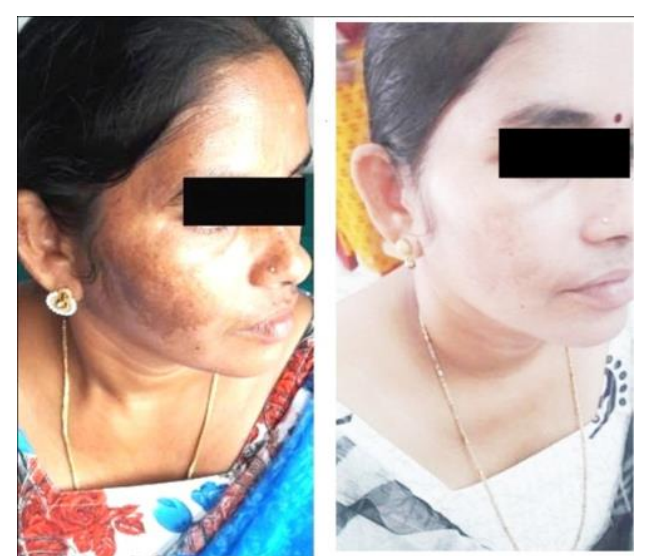

Fig. 3: Photograph showing a case of melasma on combinational therapy of SPF-1064nm-QSNdYL and $20 \%$ azelaic acid. i) week 0 (MASI 23.6) ii) week 12 (MASI 7.9)

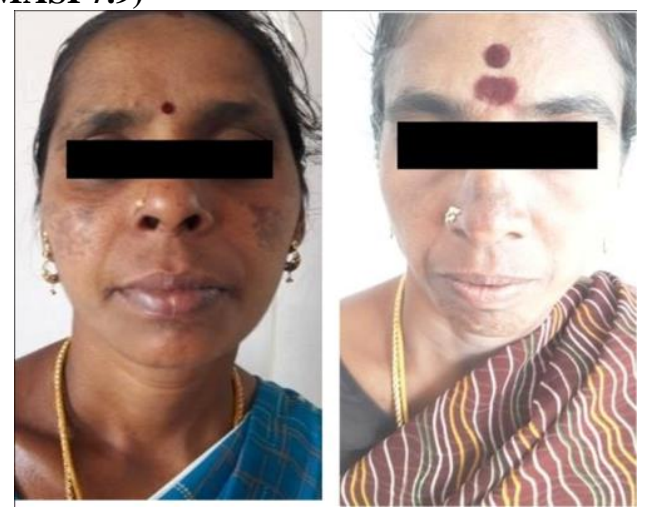

Fig. 4: Photograph showing a case of melasma on combination therapy of SPF-1064-nm-QSNdYL and $20 \%$ azelaic acid. i) week 0 (MASI 19.3) ii) week 12 (MASI 6.8) 


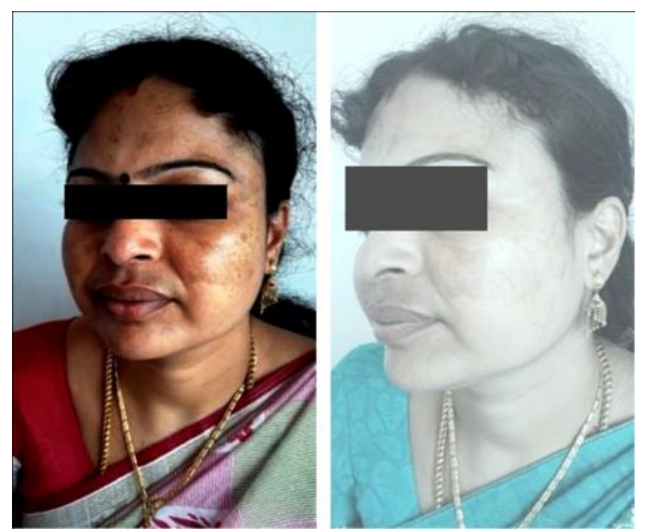

Fig. 5: Photograph showing a case of melasma on sequential therapy of Microdermabrasion followed by SPF-1064-nm-QSNdYL i) week 0 (MASI 18.4) ii) week 12 (MASI 10.7)

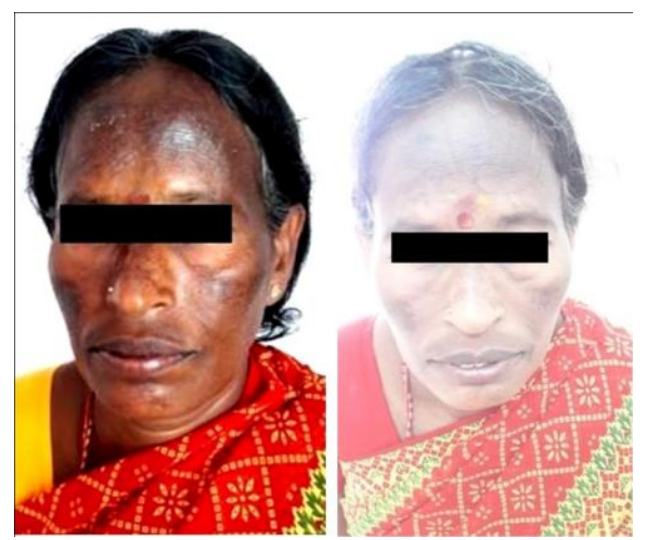

Fig. 6: Photograph showing a case of melasma on sequential therapy of Microdermabrasion followed by SPF-1064-nm-QSNdYL i) week 0 (MASI 23.2) ii) week 12 (MASI 11.8)

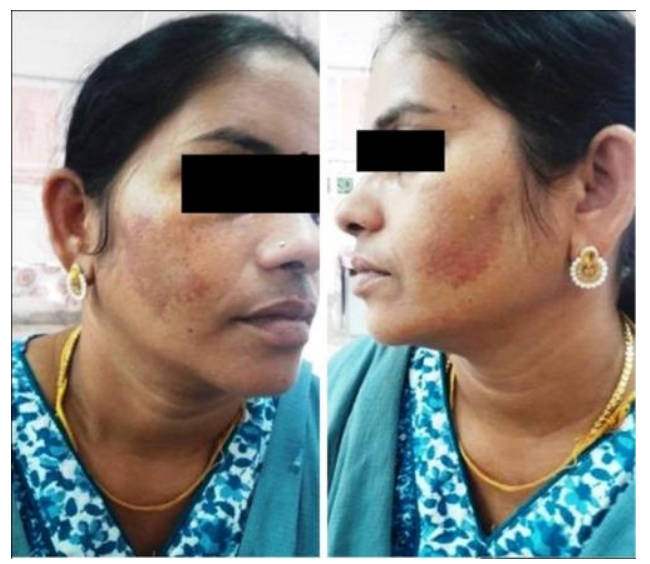

Fig. 7: Complications: Photograph showing a case of melasma with erythema after treatment with combination therapy of SPF-1064-nm-QSNdYL and $20 \%$ azelaic acid.

\section{Discussion}

Melasma is the commonest hyperpigmentary disease seen in Asians that is very difficult to manage.
In our study most subjects were of 31 to 40 years of age. The subjects mean age was 33.6 years. This is comparable to a study by Arun Achar et al., ${ }^{10}$ which was 33.45 years and another study by Griffiths et al., ${ }^{11}$ which showed 30 years. Centero facial melasma was the commonest type $(70 \%)$ noted. Next came the malar type seen in $21.66 \%$ and then the mandibular type in $8.34 \%$ of cases. This was substantiated by prior Indian research. ${ }^{12}$ Under woods lamp, epidermal type was noted in 7(11.67\%), dermal type in $12(20 \%)$ and mixed type in $41(68.33 \%)$ patients. This result is contrary to a prior research that found the epidermal type to be more common $(72 \%)$ then to follow by dermal type (22\%) and mixed type $(5 \%)$ of melasma. ${ }^{6}$ Major precipitating factor in $38(63.33 \%)$ patients was sun exposure followed by positive family history in 23 (38.33\%) patients that is comparable to a study by pathak et al., ${ }^{13}$ which suggested that UV exposure and genetics as important factors in melasma.

Although $50 \%$ of cases can achieve total clearance with topical preparations like Kligman's formula, the rate of recurrence are high. ${ }^{14}$ The gold standard depigmention medication used universally in the management of melasma is Hydroquinone (2-5\%) used alone or in combinational therapy. Due to the undesirable consequence of Hydroquinone like irritant or allergic contact eczema, rebound hyperpigmentation and confetti like depigmentaton, there is of late a surge in the use of epicutaneous azelaic acid (20\%) in the management of melasma. Azelaic acid causes inhibition of tyrosinase that specifically targets hyperactive or abnormal melanocytic cells, thereby leading to cellular toxic inhibition of DNA synthesis and mitochondrial enzymes. It neither produces leukoderma nor leads to exogenous ochronosis on prolonged use.

Of late, Laser technology is the main stay in the treatment of various pigmentation diseases. Sub photothermolytic fluence - 1064-nm Q switched Nd:YAG laser( SPF-1064-nm-QSNdYL) is primarily a pigment specific laser that emits energy in the near infra-red region of $1064 \mathrm{~nm}$ and follows the principle of selective photothermolysis, ${ }^{15}$ thereby limiting the destruction to the melanocytes leading to rupture of melanosomes and annihilation of dermal melanophages. ${ }^{16}$ Though high fluence 1064-nm QSNYL have produced better outcome in fair skinned melasma cases, an increased prevalance of complications were noted in dark skinned cases. Hence sub photothermolytic fluence - 1064-nm Q switched Nd:YAG laser( SPF-1064-nm-QSNdYL) $\left(<5 \mathrm{~J} / \mathrm{cm}^{2}\right)$ therapy delivered over a larger spot size $(6-8 \mathrm{~mm})$ leads to destruction of melanin granules with its dispersal into the cytoplasm without much cellular annihilation. By giving subsequent sub photothermolytic low fluence therapy at weekly intervals and also taking care that the sum total accumulated dosage is much lower than the total toxic accumulated energy required to destroy the cell, pigment lightening effect can be achieved. This 
mechanism of sub cellular selective photo thermolysis (SSP), without cell fragmentation or death is termed as biostimulation effect of SPF-1064-nm- QSNdYL. ${ }^{17}$

In our research with SPF-1064-nm-QSNdYL, the outcome was statistically significant with average improvement of $19.28 \%$ at 6 weeks and $39.67 \%$ at 12 weeks in the mean MASI scoring with no complications. Based on a research by Polnikorn et al., two patients of long duration dermal type melasma reported decrease of both epidermal and dermal pigmentation with Medlite C6 used once a week for a period of 10 weeks with sub photothermolytic fluence 1064-nm QSNYL $\left(<5 \mathrm{~J} / \mathrm{cm}^{2}\right)$ with no recurrences during 1 year and 6 months of follow up. ${ }^{18}$ In a study cho et al. in 2009 , reported that by using low pulse energy $1064 \mathrm{~nm}$ QSNYL in 25 cases at periods of 2 weeks apart ( $6-\mathrm{mm}$ spot size, $2.5 \mathrm{~J} / \mathrm{cm}^{2}$, two passes with proper overlap) yielded better results in the management of melasma. ${ }^{19}$ In a research Suh et al., in 2010 reported that by using $1064 \mathrm{~nm}$ QSNYL at weekly intervals for a period of 10 weeks was both safe as well as effective for the management of melasma in Asians. ${ }^{20}$ In a research by Kar et al., ${ }^{21} 25$ cases were given subthermolytic QSNYL treatment for a period of 12 weeks and this resulted in an average achievement of $47.93 \%$ which is comparable to our research which yielded an average result in mean MASI of $39.67 \%$ with no complications.

The combination therapy of daily epicutaneous $20 \%$ azelaic acid with weekly SPF-1064-nm-QSNdYL in management of melasma produces lightening of pigmentation in both epidermal and mixed type of melasma by acting synergistically. In a research by Bansal et al.,22 the combinational therapy of QSNYL with low fluence and 20\% azelaic acid showed better results with an average improvement noted in mean MASI of $46.78 \%$ and $72.75 \%$ at the end of 6 weeks and 12 weeks which is comparable to that achieved in our study with an average improvement noticed in mean MASI of $42.14 \%$ and $74.96 \%$.

The sequential therapy of Microdermabrasion followed by SPF-1064-nm-QSNdYL in the treatment of melasma is a simplified, non-invasive therapy with least complications, minimal recuperation time with long term remission that is suitable to all skin types. In a study by Arielle N.B. Kauvar., ${ }^{23} 27$ female cases of mixed type of melasma with phototypes II-V and also refractory to prior therapies showed good reponse to sequential therapy with QSNYL at sub photothermolytic fluence of $1.6-2 \mathrm{~J} / \mathrm{cm}^{2}$ with 5 or $6 \mathrm{~mm}$ spot, administered immediately after microdermabrasion. Twenty-two patients $(81 \%)$ had $>75 \%$ improvement of melasma, 11 patients (40\%) showed $>95 \%$ improvement and $>50 \%$ noted better results within a month after the initiation of therapy which is comparable to that accomplished in our research with an average improvement at the end of 6 weeks and 12 weeks in mean MASI of $30.22 \%$ and $43.97 \%$.

In our research, in epidermal melasma type, the maximum reduction in mean MASI scoring of $74.57 \%$ at the end of 12 weeks was noted in combinational group (SPF-1064-nm-QSNdYL and epicutaneous 20\% azelaic acid), mostly due to the their synergistic mechanism. It was followed by mean MASI score reduction of $41.59 \%$ in sequential group (microdermabrasion immediately followed by SPF1064-nm- QSNdYL) and 38.68\% in the SPF-1064-nm QSNdYL alone group. On comparison of the three groups, the mean MASI score reduction in dermal melasma, for combination group and sequential group were almost similar with results of $70.30 \%$ and $61.26 \%$ followed by $45.89 \%$ in the SPF-1064-nm QSNdYL alone group. We postulate that in addition to the selective action of low-fluence 1064nm QSNYL on the dermal melanosomal rupture and annihilation of melanophages, there is an additional impact on epidermal melanosmes by combination therapy and sequential therapy thereby leading to a further decrease in mean MASI scoring when compared to SPF-1064$\mathrm{nm}$ QSNdYL therapy alone in dermal type of melasma. In mixed type of melasma, the maximum decrease in mean MASI scoring of $75.91 \%$ at 12 weeks was noted in combination therapy, followed by $46.78 \%$ in sequential therapy and the least of $43.74 \%$ with SPF1064-nm QSNdYL alone group. This may be due to the synergistic mechanism of both epicutaneous azelaic acid along with SPF-1064-nm QSNdYL.

In our study, we observed immediate complications like erythema and mild burning sensation in both combination and sequential groups that subsided within an hour in all the patients. Late onset hyperpigmentation was noted in sequential group. No complications were noted in SPF-1064-nm- QSNdYL alone group.

\section{Conclusion}

This study proves the superior efficacy of combinational therapy of SPF-1064-nm- QSNdYL with azelaic acid in epidermal, dermal and mixed types of melasma with minimal adverse effects followed by sequential therapy of microdermabrasion with SPF1064-nm- QSNdYL and SPF-1064-nm- QSNdYL alone therapy. Further research is needed to substantiate this potential option in the management of melasma.

Funding: No funding sources. Conflict of interest: None declared.

\section{References}

1. Grimes PE. Melasma: Etiologic and therapeutic considerations. Arch Dermatol 1995;131:1453-47. [PubMed: 7492140]

2. Pasricha JS, Khaitan BK, Dash S. Pigmentary disorders in India. Dermatol Clin 2007;25:343-52. [PubMed: $17662900]$ 
3. Kim EH, Kim YC, Lee ES, Kang HY. The vascular factors of melasma. J Dermatol Sci 2007;46:111-6. [PubMed: 17363223]

4. Pérez M, Sánchez JL, Aguiló F. Endocrinologic profile of patients with idiopathic melasma. J Invest Dermatol 1983;81:543-45. [PubMed: 6644096]

5. Grimes PE, Yamada N, Bhawan J. Light microscopic, immunohistochemical and ultrastructural alteration in patients with melasma. Am J Dermatopathol 2005;27:96101. [PubMed: 15798432]

6. Sanchez NP, Pathak MA, Sato S, Fitzpatrick TB, Sanchez JL, Mihm MC., Jr Melasma: A clinical, light microscopic, ultra structural, and immunofluorescence study. J Am Acad Dermatol 1981;4:698-710. [PubMed: 6787100]

7. Kang WH, Yoon KH, Lee ES, Kim J, Lee KB, Yim H, et al. Melasma: Histopathological characteristics in 56 Korean patients. Br J Dermatol 2002;146:228-37. [PubMed: 11903232]

8. Lipper GM, Anderson RR. Lasers in dermatology. In: Freedburg IM, Eisen AZ, Ketal W, editors. Fitzpatrick dermatology in general medicine. 7th ed. New York: McGraw Hill; 2007. p. 2274.

9. Pandya A, Berneburg M, Ortonne JP, Picardo M. Guidelines for clinical trials in melasma: Pigmentation disorders academy. Br J Dermatol 2006;156:21-8. [PubMed: 17176301]

10. Achar A, Rathi SK. Melasma: a clinicoepidemiological study of 312 cases. Indian J Dermatol 2011;56(4):38082.

11. Griffiths CE, Finkel LJ, Ditre CM, Hamilton TA, Ellis $\mathrm{CN}$, Voorhees JJ. Topical tretinoin (retinoic acid) improves melasma. A vehicle controlled, clinical trial. $\mathrm{Br}$ J Dermatol 1993;129:415-21. [PubMed: 8217756]

12. Javaheri SM, Handa S, Kaur I, Kumar B. Safety and efficacy of glycolic acid facial peel in Indian women with melasma. Int J Dermatol 2001;40:354-7. [PubMed: 11555002]

13. Pathak MA, Fitzpatrick TB, Kraus EW. Usefulness of retinoic acud in the treatment of melasma. J Am Acad Dermatol 1986;15:894-99.

14. Gupta AK, Gover MD, Nouri K, Taylor S. The treatment of melasma: A review of clinical trials. J Am Acad Dermatol 2006;55:1048-65.

15. Anderson RR, Parrish JA. Selective photothermolysis.
Precise microsurgery by selective absorption of pulsed radiation. Sci 1983;220:524-7. [PubMed: 6836297]

16. Mun JY, Jeong SY, Kim JH, Han SS, Kim IH. A low fluence Q-switched Nd: YAG laser modifies the 3D structure of melanocyte and ultrastructure of melanosome by subcellular-selective photothermolysis. J Electron Microsc (Tokyo) 2011;60:11-8. [PubMed: 20937709]

17. Wattanakrai P, Mornchan R, Eimpunth S. Low-fluence Q-switched neodymium-doped yttrium aluminum garnet $(1,064 \mathrm{~nm})$ laser for the treatment of facial melasma in Asians. Dermatol Surg 2010;36:76-87.

18. Polnikorn N. Treatment of refractory dermal melasma with the MedLite C6 Q-switched Nd: YAG laser: Two case reports. J Cosmet Laser Ther 2008;10:167-73. [PubMed: 18788035]

19. Cho SB, Kim JS, Kim MJ. Melasma treatment in Korean women using a 1064-nm Q-switched Nd: YAG laser with low pulse energy. Clin Exp Dermatol 2009;34:e847-50. [PubMed: 19817757]

20. Suh KS, Sung JY, Roh HJ, Jeon YS, Kim YC, Kim ST. Efficacy of $1064 \mathrm{~nm}$ Q switched Nd:YAG Laser in melasma. J Dermatolog Treat 2011;22:233-8.

21. Kar HK, Gupta L, Chauhan A. A comparative study on efficacy of high and low fluence Q-switched Nd: YAG laser and glycolic acid peel in melasma. Indian $J$ Dermatol Venereol Leprol 2012;78:165-71. [PubMed: 22421647]

22. Charu Bansal, Hira Naik, Hemanta K Kar, and Amrita Chauhan. A Comparison of Low-Fluence 1064-nm QSwitched Nd: YAG Laser with Topical 20\% Azelaic Acid Cream and their Combination in Melasma in Indian Patients. J Cutan Aesthet Surg 2012; 5(4): 266-272.

23. Kauvar AN. Successful treatment of melisma using a combination of microderm and Q-switched Nd:YAG lasers. Laser Surg Med 2012;44(2):117-24.

How to cite this article: Daniel S., Kumar R., Geetha K. A comparative study of efficacy of 1064-nm Qswitched Neodymium: YAG laser at sub photothermolytic fluence alone and in combination therapy with epicutaneous $20 \%$ azelaic acid and in sequential therapy with microdermabrasion in melasma in South India. Indian $J$ Clin Exp Dermatol 2018;4(4):306-13. 